\title{
Evaluation of image-enhanced endoscopic technology using advanced diagnostic endoscopy for the detection of early gastric cancer: a pilot study
}

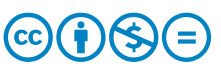

\author{
Authors \\ Yamamichi², Kazuhiko Koike $^{2}$ \\ Institutions \\ 1 Department of Endoscopy and Endoscopic Surgery, \\ Graduate School of Medicine, The University of Tokyo, \\ Tokyo, Japan \\ 2 Department of Gastroenterology, Graduate School of \\ Medicine, The University of Tokyo, Tokyo, Japan \\ 3 Center for Epidemiology and Preventive Medicine, \\ Graduate School of Medicine, The University of Tokyo, \\ Tokyo, Japan
}

Daisuke Yamaguchi ${ }^{1}$, Shinya Kodashima ${ }^{2}$, Mitsuhiro Fujishiro ${ }^{1,2}$, Satoshi Ono ${ }^{2}$, Keiko Niimi²,3, Satoshi Mochizuki², Yosuke Tsuji ${ }^{2}$, Itsuko Asada-Hirayama², Yoshiki Sakaguchi², Satoki Shichijo², Chihiro Minatsuki², Nobutake

submitted 25.7.2016

accepted after revision 24.5.2017

Bibliography

DOI https://doi.org/10.1055/s-0043-113632 |

Endoscopy International Open 2017; 05: E825-E833

(c) Georg Thieme Verlag KG Stuttgart · New York

ISSN 2364-3722

Corresponding author

Shinya Kodashima, Department of Gastroenterology, Graduate School of Medicine, The University of Tokyo, 7-3-1 Hongo, Bunkyo-ku, Tokyo 113-8655, Japan

Fax: +81-3-5800-9015

kodashima-tky@umin.ac.jp

\section{ABSTRACT}

Background and study aims Image-enhanced endoscopy (IEE) plays an important role in early detection and detailed examination of early gastric cancer (EGC). The current study aimed to clarify the efficacy of IEE using advanced diagnostic endoscopy for EGC detection without magnification.

Patients and methods We performed endoscopic examinations without magnification in patients referred to our hospital with a diagnosis of upper gastrointestinal tumor detected through routine screening endoscopy. In this study, we used three IEE technologies: narrow-band imaging; blue laser imaging; and i-scan optical enhancement. The detection rates for EGC between IEE and white-light imaging (WLI) were compared.

Results Between July 2013 and June 2014, 156 patients were enrolled. Among upper gastrointestinal tumors, we analyzed endoscopic examination results of 119 lesions that were histologically diagnosed as EGC in 109 patients. The EGC detection rate in the IEE plus WLI groups was $77.3 \%$. Although the EGC detection rate in the IEE group was higher than that in the WLI group ( $80.0 \%$ vs. $70.3 \%$ ), there was no significant difference between these two modalities. An important detection factor using IEE was tumor circumference, where the rate of detection in the anterior wall and lesser curvature was significantly higher than that in the posterior wall and greater curvature $(P=0.046)$. An important detection factor using WLI was color variation, where the rate of occurrence of a reddened or pale tumor was significantly higher than that of normal colored tumors $(P=0.030)$.

Conclusions The detection rate of EGC without magnification was similar between the IEE group and the WLI group. Important detection factors differed between IEE and WLI; therefore, the IEE and WLI modalities have different characteristics regarding EGC detection. Consequently, we propose to use both IEE and WLI in the evaluation of EGC.

\section{Introduction}

Gastric cancer is the third leading cause of death from cancer death worldwide [1]. Early detection and therapy for gastric cancer can improve the 5-year survival rate to $96 \%$; however, there is a high mortality rate for advanced gastric cancer [2].
Therefore, early diagnosis of cancer is important in the management of gastric cancer.

White-light imaging (WLI) has been used as the standard endoscopic examination for identification of suspicious lesions, but it is difficult to make an accurate diagnosis of early gastric cancer (EGC) using only WLI. Several studies have indicated 

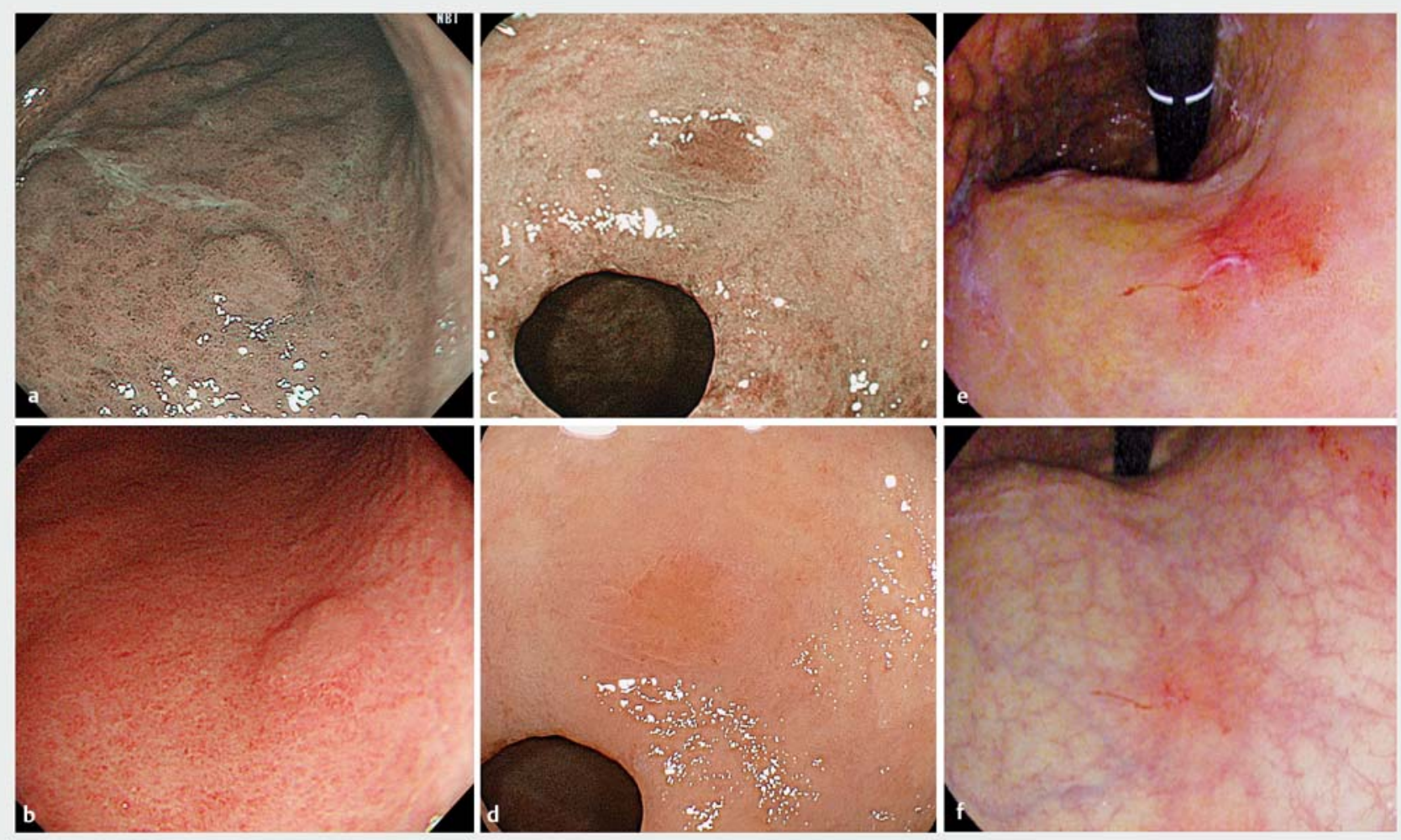

- Fig. 1 Endoscopic images of EGCs in the three IEE systems (NBI, BLI, and i-scan OE) and WLI. a EGC with NBI. b EGC with WLI using the EVIS LUCERA ELITE system. $\mathbf{c}$ EGC with BLI. d EGC with WLI using the LASEREO system. e EGC with i-scan OE. f EGC with WLI using the EPK-i 7000 system.

that sensitivity of WLI in diagnosis of superficial EGC varies from $33 \%$ to $75 \%[3-5]$.

Image-enhanced endoscopy (IEE) is a technology that has been developed to improve diagnostic ability [6]. IEE is mainly subdivided into digital, optical-digital, and chromoendoscopy modalities; efficacy of optical-digital methods for the detection and identification of gastrointestinal tumors has been frequently reported in recent years [7].

Narrow band imaging (NBI), which is classified as an opticaldigital method [8], is an advanced endoscopic imaging technology that has recently been developed, in which spectral bandwidth filters are used to improve the accuracy of diagnosis [9]. Although endoscopy using NBI is remarkably useful for differential diagnosis of superficial gastric lesions that are identified under white light $[3,5,10]$, there seems to be very little evidence to justify routine use of $\mathrm{NBI}$ during routine screening endoscopy; that is because of the poor light intensity generated, which causes the image to appear darker especially in the stomach and colon. Newer-generation NBI processors (290 and 190 series) with a higher light intensity are currently being launched commercially and studies using these processors are eagerly awaited [11]. NBI processors with higher light intensities have been developed and may potentially improve detection rates. Next-generation NBI colonoscopy represents a significant improvement in detection of colonic polyps [12].
Blue laser imaging (BLI) and i-scan optical enhancement (OE) are also classified as optical-digital methods. BLI uses a laser source with a short wavelength, and i-scan OE uses optical filters that limit the spectral characteristics of the illumination light to improve endoscopic observation of microsurface structures and microvascular patterns in superficial mucosa [13]. BLI and i-scan OE have an observational mode with high-illumination intensity, namely BLI-bright mode and i-scan OE mode 2, respectively; these modes may be promising developments not only for characterization but also for screening of gastrointestinal tumors, because they can maintain high-illumination intensity [14-16]. Thus, recently developed IEE systems ensure the illumination intensity for wide-range observation of the fully extended gastrointestinal lumen using high light intensity. Therefore, they are expected to also be useful for detection and identification of EGC without magnification.

However, the effectiveness of the 3 IEE systems (NBI, BLI, and $\mathrm{i}$-scan $\mathrm{OE}$ ) in improving the detection rate for $\mathrm{EGC}$ in screening endoscopy without magnification has not yet been systematically evaluated. In the current study, we conducted a diagnostic trial using prospectively collected data involving the 3 IEE systems regarding the EGC detection rate. We also investigated the characteristics of factors associated with the detection of EGC using IEE and WLI. 


\section{Patients and methods}

\section{Study subjects}

This was a single-center prospective pilot diagnostic study. Patients who had been previously diagnosed with upper gastrointestinal tumors using screening endoscopy at our hospital or other hospitals between July 2013 and June 2014 were enrolled. Written informed consent was obtained from patients who met the inclusion criteria prior to study enrollment. We excluded patients who refused to provide consent prior to examination. Patients who had been diagnosed with advanced gastric cancer were excluded. The target sample size was set at 100 patients, a number achievable within 1 year.

The study was initiated after approval by the Medical Ethics Committee of the University of Tokyo Hospital and registration in the University Hospital Medical Network Clinical Trial Registry (UMIN000011139) on July 7, 2013.

\section{Endoscopy system and endoscopic procedure}

Endoscopic examinations using WLI and IEE were performed using 1 of 3 video-endoscopy systems: the EVIS LUCERA ELITE system (Olympus Medical Systems, Tokyo, Japan); the LASEREO system (Fujifilm Medical Co. Ltd., Tokyo, Japan); and the EPK-i 7000 system (HOYA Corporation, PENTAX Lifecare Division, Tokyo, Japan). The 3 video-endoscopy systems were equipped with each IEE technology, which was classified as the opticaldigital method as follows: NBI for the EVIS LUCERA ELITE system; BLI for the LASEREO system; and i-scan OE for the EPK-i 7000 system.

The NBI system allows for narrow-band observation using the original optical filters, and light in the blue to green region is well absorbed by hemoglobin, providing good visualization of capillaries and vessels in the superficial mucosa [17-19]. In the current study, we used newer-generation NBI processors (i.e., the EVIS LUCERA ELITE system).

The BLI system has a unique illumination setup involving 2 lasers and a white light phosphor, and we achieved clear and detailed visualization of microsurface structures and microvascular patterns of the superficial mucosa $[14,15,20]$. We used the BLI-bright mode in this study.

In the i-scan OE mode 2, the main wavelengths of the short and mid-wavelengths correspond to the peaks of the hemoglobin absorption spectrum, and light between these peaks is also continuously emitted at low intensity by raising baseline transmittance to achieve the maximum amount of illumination [21]. We used the i-scan OE system in mode 2.

Representative endoscopic images are shown in > Fig. 1.

\section{Study design}

We performed diagnostic endoscopy for detection of EGC regarding the primary modality, using WLI followed by IEE (WLI group) or IEE followed by WLI (IEE group). Patients were randomly assigned to 6 groups as follows: O-IEE group (NBI followed by WLI); O-WLI group (WLI followed by NBI); F-IEE group (BLI followed by WLI); F-WLI group (WLI followed by BLI); H-IEE group (i-scan OE followed by WLI); and H-WLI group (WLI fol-
Assessment for enrollment 162 patients

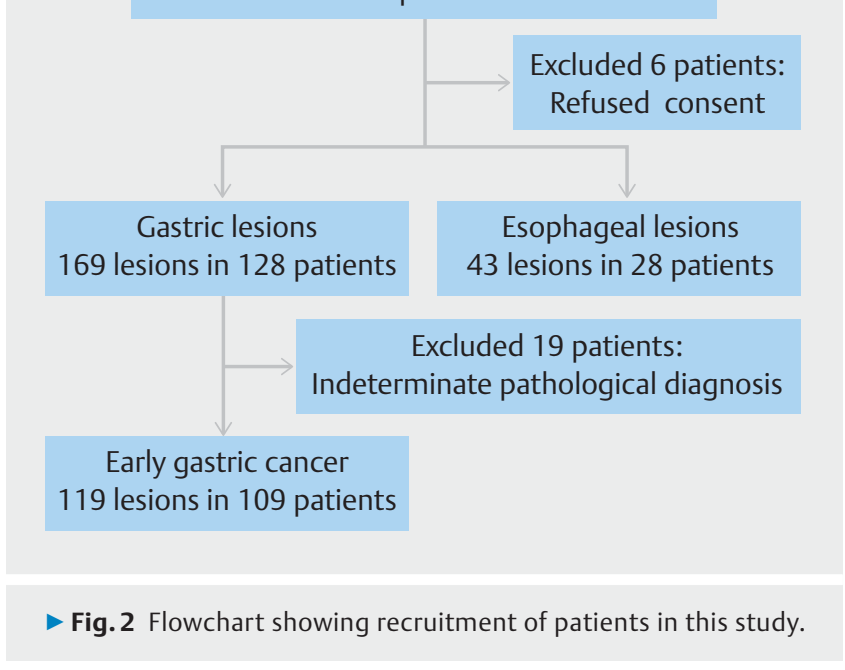

lowed by i-scan OE). The subjects were assigned by the envelope method just before the endoscopic observation. All endoscopic observations were performed by an endoscopist who had no information concerning the EGC patients (size, location, and numbers) but had only information on presence of EGC, and by an assistant endoscopist who had access to all previous information. After the observation using the primary modality was completed, the assistant endoscopist immediately recorded results on the case record form (CRF). After completion of the CRF for the primary modality, a second observation using the secondary modality was performed and the results were recorded on the CRF. Examination time was defined as the first observation time of endoscopic screening procedure for EGC using IEE or WLI. In this study, when endoscopists detected a suspected lesion, they were classified as EGC. Successful detection of EGC was defined as the agreement between detection of EGC/suspected EGC and the histological diagnosis of biopsy specimen after endoscopic screening procedure.

We compared the EGC detection rate between IEE and WLI groups. In addition, we evaluated the EGC detection rate in IEE plus WLI groups. The detection rate in IEE plus WLI groups was defined as the total detection rate of the first and second observation using IEE and WLI.

We also investigated characteristics of detection factors (tumor size, location, and circumference; color variation using WLI, macroscopic classification; histological classification; and depth of invasion) in IEE and WLI groups. Color variations concerning EGC in the WLI system were defined as normal and different (reddened/pale). And we investigated 5 cases that could not be diagnosed using either IEE or WLI for the details.

In this study, 10 endoscopists (S. K., M. F., S. O., K. N., S. M., Y.T., I. H., Y.S., S.S., C. M.) performed the procedures for diagnosis of EGC using each of the 3 IEE modalities. All endoscopists had experience with $>40$ cases of endoscopic submucosal dissection and $>100$ cases of diagnosis of EGC using IEE such as 
- Table 1 Patients characteristics.

\begin{tabular}{|c|c|c|c|c|}
\hline & $\mathbf{n}$ & IEE group & WLI group & $P$ value \\
\hline Number of patients & 109 & 50 & 59 & \\
\hline Median age (years) & $70.3 \pm 10.8$ & $69.7 \pm 10.3$ & $70.8 \pm 11.2$ & 0.582 \\
\hline Sex & & & & 0.780 \\
\hline - Male & 95 & 43 & 52 & \\
\hline - Female & 14 & 7 & 7 & \\
\hline Number of tumor lesions & 119 & 55 & 64 & \\
\hline Examination time (min) & $5.9 \pm 2.0$ & $5.7 \pm 2.1$ & $6.1 \pm 2.0$ & 0.312 \\
\hline Tumor size (mm) & $16.8 \pm 13.2$ & $17.4 \pm 11.3$ & $16.3 \pm 14.8$ & 0.654 \\
\hline Tumor lesion & & & & 0.805 \\
\hline - Upper third & 18 & 8 & 10 & \\
\hline - Middle third & 55 & 24 & 31 & \\
\hline - Lower third & 46 & 23 & 23 & \\
\hline Tumor location & & & & 0.913 \\
\hline - Anterior wall & 18 & 10 & 8 & \\
\hline - Posterior wall & 27 & 27 & 13 & \\
\hline - Lesser curvature & 47 & 24 & 23 & \\
\hline - Greater curvature & 27 & 16 & 11 & \\
\hline Color variation & & & & 0.202 \\
\hline - Reddening & 70 & 35 & 35 & \\
\hline - Pale & 21 & 6 & 15 & \\
\hline - Normal & 28 & 14 & 14 & \\
\hline Macroscopic classification & & & & 0.298 \\
\hline - Elevated type (0-IIa) & 33 & 19 & 24 & \\
\hline - Flat type (0-IIb) & 2 & 1 & 1 & \\
\hline - Depressed type (0-IIc) & 84 & 35 & 49 & \\
\hline Histological classification & & & & 0.236 \\
\hline - Intestinal type & 106 & 51 & 55 & \\
\hline - Diffuse type & 3 & 0 & 3 & \\
\hline - Mixed type & 10 & 4 & 6 & \\
\hline Depth of invasion & & & & 0.782 \\
\hline . m & 97 & 46 & 51 & \\
\hline . $\mathrm{sm} 1$ & 10 & 3 & 7 & \\
\hline - $\mathrm{sm} 2$ & 12 & 6 & 6 & \\
\hline
\end{tabular}

$\mathrm{NBI}, \mathrm{BLI}$ and i-scan OE prior to this study. This experience reduced the effect of the learning curve on the analysis.

\section{Statistical analysis}

We compared the EGC detection rate between IEE and WLI groups, and the characteristics of detection factors in these groups using the $x^{2}$ test and Student's $t$-test. Univariable logistic regression analysis was performed to examine characteristics of detection factors adjusted for each of the other tumor factors. Multivariable logistic regression analyses were performed using all tumor factors (tumor size, tumor lesion, tumor location, color variation, macroscopic classification, histologi- 


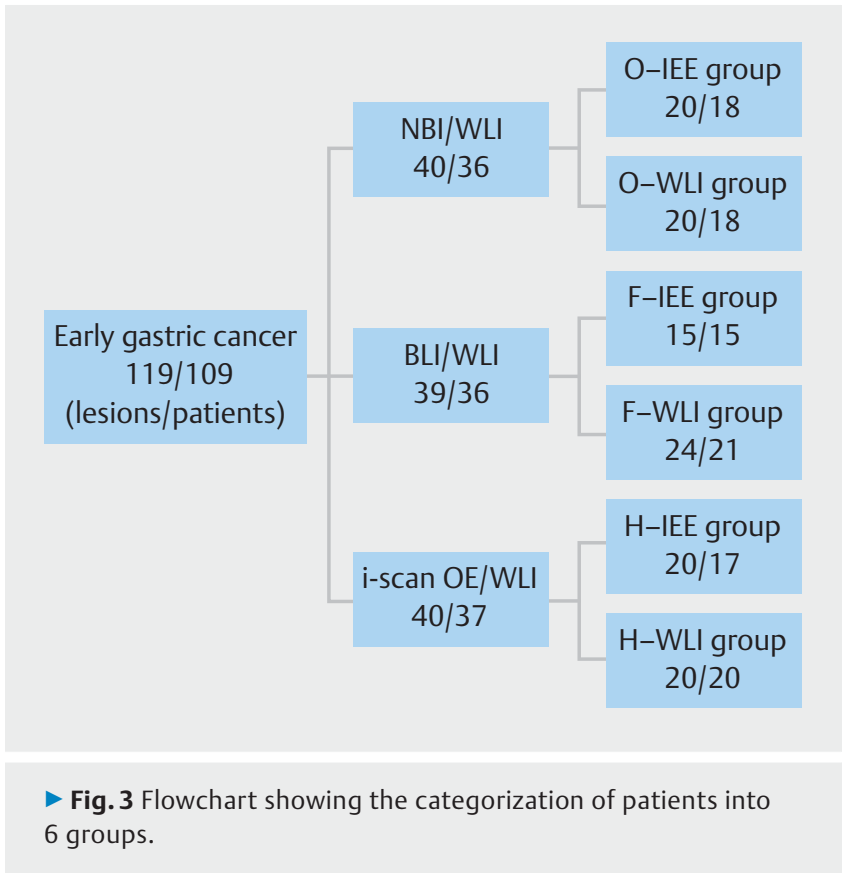

cal classification and depth of invasion) as independent variables simultaneously. Odds ratios and $95 \%$ confidential intervals $(\mathrm{Cl})$ were determined by these uni- and multivariable logistic regression models. We also examined some cases of EGC that were only detected using either IEE or WLI. A 2-tailed $P$ value $<0.05$ was considered to be statistically significant in all analyses. All statistical analyses were performed using JMP version 9.0.2 (SAS Institute, Tokyo, Japan).

\section{Results}

Between July 2013 and June 2014, 162 patients diagnosed with early-stage tumors after previous endoscopic examinations were enrolled in this study. We excluded 6 patients who refused to give consent prior to examination. Additionally, 43 lesions in 28 patients with esophageal lesions were excluded. Lesions that were histologically diagnosed as adenomas (6) and advanced gastric cancers (9) were excluded from the study. In addition, small lesions that were pathologically diagnosed as noncancerous after examination of biopsy specimens were excluded from the study. In total, 119 lesions in 109 patients were included in this study ( $>$ Fig. 2 ).

- Table 1 shows characteristics of patients who had EGC detected in the IEE and WLI groups. Ninety-five patients (87.2\%) were male; mean $( \pm S D)$ patient age was $70.3 \pm 10.8$ years. Examination time $( \pm S D)$ was $6.1 \pm 2.0$ minutes. There was no significant difference between the IEE and WLI groups. Mean $( \pm$ SD) EGC size was $16.8 \pm 13.2 \mathrm{~mm}$. EGCs were mainly located in the middle third of 55 lesions (46.2\%) and in the lesser curvature of 47 lesions (39.5\%). Using the gastric cancer macroscopic classification, $84(70.5 \%)$ lesions were primarily classified as type 0 -Ilc. A total of 106 (89.1\%) lesions were histologically classified as being of the intestinal type. According to depth of invasion, 97 lesions (81.5\%) were intramucosal can-
- Table 2 Early gastric cancer detection rate.

\begin{tabular}{|l|l|l|l|l|}
\hline & \multicolumn{3}{|c|}{ Tumor findings } \\
\hline & $\mathbf{n}$ & $\begin{array}{l}\text { First } \\
\text { modality }\end{array}$ & $\begin{array}{l}\text { Detection rate of } \\
\text { first modality (\%) }\end{array}$ & P value \\
\hline O-IEE & 20 & 16 & 80.0 & 0.301 \\
\hline O-WLI & 20 & 12 & 60.0 & \\
\hline F-IEE & 15 & 13 & 86.7 & 0.686 \\
\hline F-WLI & 24 & 19 & 79.2 & 1.000 \\
\hline H-IEE & 20 & 15 & 75.0 & \\
\hline H-WLI & 20 & 14 & 70.0 & 0.291 \\
\hline IEE group & 55 & 44 & 80.0 & \\
\hline WLI group & 64 & 45 & 70.3 & \\
\hline
\end{tabular}

cers, and 10 lesions (8.4\%) were submucosal cancers invading to a depth of $<500 \mathrm{~mm}$. Twelve lesions $(10.1 \%)$ were submucosal cancers invading to a depth of $>500 \mathrm{~mm}$.

Regarding color variation in the WLI group, 70 lesions (58.9\%) were defined as reddened. There were no differences in sex, age, tumor size and location, macroscopic and histological classification, depth of invasion or color variations between IEE and WLI groups.

Finally, we categorized the lesions according to the 6 groups as follows: 20 lesions in O-IEE group; 20 lesions in O-WLI group; 15 lesions in F-IEE group; 24 lesions in F-WLI group; 20 lesions in $\mathrm{H}$-IEE group; and 20 lesions in $\mathrm{H}$-WLI group ( $\mathbf{F i g}$. 3).

The EGC detection rate is detailed in $>$ Table 2 . It was $77.3 \%$ in the IEE plus WLI groups. Although the rate was higher in the IEE group than in the WLI group (80.0\% vs. $70.3 \%)$, there was no significant differences between these 2 modalities. Among the 6 groups, the detection rate was $80.0 \%$ in the O-IEE group, $60.0 \%$ in the O-WLI group, $86.7 \%$ in the F-IEE group, $79.2 \%$ in the F-WLI group, $75.0 \%$ in the H-IEE group, and $70.0 \%$ in the $\mathrm{H}-\mathrm{WLI}$ group; there was also no significant difference between these 2 modalities using each endoscopic system.

Characteristics of detection factors between IEE and WLI groups are compared in $>$ Table 3 . We analyzed the OR and $95 \% \mathrm{Cl}$ for detection of tumor estimating uni- and multivariable logistic regression analysis in IEE and WLI, respectively. When using IEE methods, tumor circumference was significantly associated with tumor detection; the rate of detection of a tumor in the anterior wall and lesser curvature was significantly higher than that in the posterior wall and greater curvature, as determined using univariable logistic regression analysis (OR 7.250, $95 \% \mathrm{Cl} 1.370-38.300, P=0.020)$. This association was remained even after adjustment for each of the other tumor factors (OR 6.700, 95\% Cl 1.140-39.400, $P=0.035$ ). Similarly, in WLI group, concerning color variation, a reddened or pale tumor was detected significantly more frequently than a normal color tumor determined (odds ratio 4.000, $95 \% \mathrm{Cl} 1.140-$ 14.000, $P=0.031$ ) using univariate analysis. Using multivariable logistic regression analysis, color variation was demonstrated 
- Table 3 Comparison between IEE and WLI systems for the detection of early gastric cancer.

\begin{tabular}{|c|c|c|c|c|c|c|}
\hline & \multicolumn{6}{|l|}{ IEE group } \\
\hline & \multicolumn{3}{|l|}{ Univariable } & \multicolumn{3}{|c|}{ Multivariable } \\
\hline & Odds ratio & $95 \% \mathrm{Cl}$ & $P$ value & Odds ratio & $95 \% \mathrm{Cl}$ & $P$ value \\
\hline \multicolumn{7}{|l|}{ Tumor size (mm) } \\
\hline - $1-20$ & 0.246 & $0.028-2.130$ & 0.203 & 0.236 & $0.021-2.620$ & 0.240 \\
\hline \multicolumn{7}{|l|}{ Tumor lesion } \\
\hline - Upper and middle third & 0.536 & $0.123-2.340$ & 0.407 & 0.509 & $0.097-2.670$ & 0.425 \\
\hline \multicolumn{7}{|l|}{ Tumor location } \\
\hline - AW and LC & 7.250 & $1.370-38.300$ & 0.020 & 6.700 & $1.140-39.400$ & 0.035 \\
\hline \multicolumn{7}{|l|}{ Color variation } \\
\hline - Reddening and pale & 0.274 & $0.031-2.380$ & 0.240 & 0.320 & $0.031-3.320$ & 0.340 \\
\hline \multicolumn{7}{|l|}{ Macroscopic classification } \\
\hline - Elevated type (0-Ila) & 2.430 & $0.461-12.800$ & 0.296 & 1.980 & $0.293-13.400$ & 0.482 \\
\hline \multicolumn{7}{|l|}{ Histological classification } \\
\hline - Intestinal type & 1.560 & $0.145-16.700$ & 0.715 & 4.280 & $0.095-192.000$ & 0.454 \\
\hline \multicolumn{7}{|l|}{ Depth of invasion } \\
\hline \multirow[t]{4}{*}{ - m } & 1.360 & $0.237-7.780$ & 0.732 & 1.010 & $0.133-7.700$ & 0.990 \\
\hline & \multicolumn{6}{|l|}{ WLI group } \\
\hline & \multicolumn{3}{|l|}{ Univariable } & \multicolumn{3}{|c|}{ Multivariable } \\
\hline & Odds ratio & $95 \% \mathrm{Cl}$ & $P$ value & Odds ratio & $95 \% \mathrm{Cl}$ & $P$ value \\
\hline \multicolumn{7}{|l|}{ Tumor size (mm) } \\
\hline . $1-20$ & 0.147 & $0.018-1.220$ & 0.076 & 0.129 & $0.011-1,460$ & 0.098 \\
\hline \multicolumn{7}{|l|}{ Tumor lesion } \\
\hline - Upper and middle third & 0.453 & $0.128-1.600$ & 0.220 & 0.444 & $0.112-1.770$ & 0.249 \\
\hline \multicolumn{7}{|l|}{ Tumor location } \\
\hline - AW and LC & 1.010 & $0.332-3.070$ & 0.986 & 0.879 & $0.238-3.240$ & 0.846 \\
\hline \multicolumn{7}{|l|}{ Color variation } \\
\hline - Reddening and pale & 4.000 & $1.140-14.000$ & 0.031 & 4.510 & $1.050-19.300$ & 0.042 \\
\hline \multicolumn{7}{|l|}{ Macroscopic classification } \\
\hline - Elevated type (0-Ila) & 2.570 & $0.512-12.900$ & 0.252 & 1.570 & $0.241-10.300$ & 0.636 \\
\hline \multicolumn{7}{|l|}{ Histological classification } \\
\hline - Intestinal type & 0.305 & $0.035-2.640$ & 0.281 & 0.748 & $0.061-9.140$ & 0.820 \\
\hline \multicolumn{7}{|l|}{ Depth of invasion } \\
\hline . m & 0.436 & $0.086-2.210$ & 0.316 & 0.845 & $0.134-5.320$ & 0.858 \\
\hline
\end{tabular}

to be an important detection factor concerning WLI independently (odds ratio 4.510; $95 \% \mathrm{Cl}, 1.050-19.300 ; P=0.042$ ).

In $>$ Table 4, 5 cases involving detection of EGC using either IEE or WLI are presented. Three EGC cases were only detected using IEE, and all of these ECGs were located in the lesser curva- ture. Two EGC cases were found exclusively using WLI, and in both cases color variation involved reddened lesions. Representative endoscopic pictures of Case 3 and Case 5 are shown in $>$ Fig. 4 and $\triangleright$ Fig. 5, respectively. 
- Table4 Cases involving detection of early gastric cancer using either the IEE or WLI.

\begin{tabular}{|l|l|l|l|l|l|l|l|}
\hline & $\begin{array}{l}\text { Finding } \\
\text { modality }\end{array}$ & $\begin{array}{l}\text { Tumor } \\
\text { size }\end{array}$ & $\begin{array}{l}\text { Tumor } \\
\text { location }\end{array}$ & $\begin{array}{l}\text { Tumor } \\
\text { circumference }\end{array}$ & $\begin{array}{l}\text { Color } \\
\text { variation }\end{array}$ & $\begin{array}{l}\text { Macroscopic } \\
\text { classification }\end{array}$ & $\begin{array}{l}\text { Histological } \\
\text { classification }\end{array}$ \\
\hline Case 1 & O-IEE & 5 & U & LC & Normal & -IlC & Intestinal \\
\hline invasion
\end{tabular}

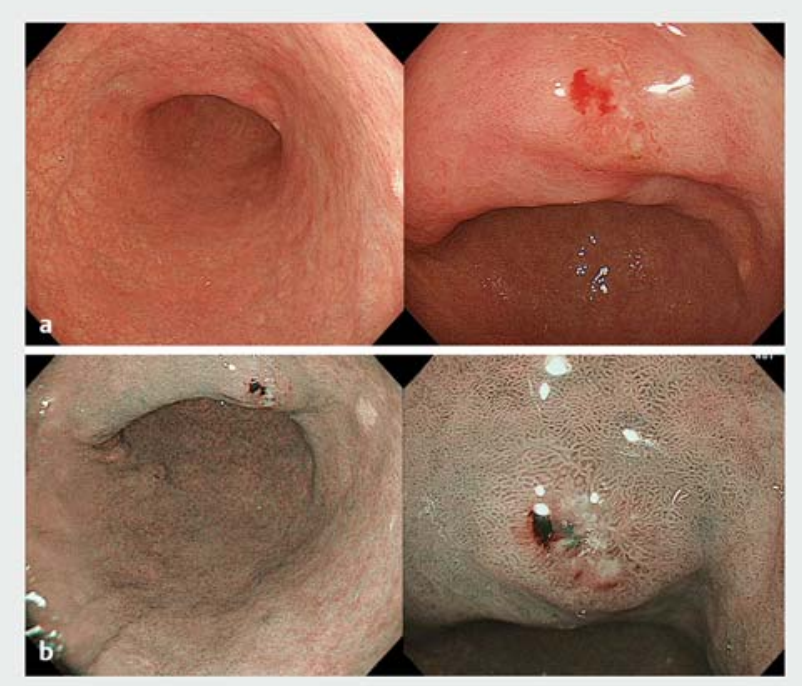

- Fig. 4 Endoscopic images from Case 3. EGC was located in the lesser curvature of the lower part of the gastric body with WLI a and IEE $\mathbf{b}$. The lesion was detected as erosion with WLI a but was observed as EGC with IEE $\mathbf{b}$ because of higher contrast and irregular vascular pattern between the cancer and surrounding tissue.

\section{Discussion}

The NBI, BLI and i-scan OE were developed as each new IEE technology for the early detection and detailed examination of EGC. These 3 modalities were classified the optical-digital methods, and considered with the same effect as IEE technology in clinical fields. Therefore, we categorized among 6 groups without a difference of IEE in this study.

IEE has been designed to improve visualization of microvascular and mucosal patterns in the gastrointestinal tract. The usefulness of magnifying endoscopy involving IEE methods for detection and diagnosis of EGC has been reported [22,23]. These methods appear to constitute a promising modality for improving accuracy of diagnosing EGC. The IEE system was expected to be useful for detection of EGC. However, in our study, there was no significant difference in EGC detection rate between WLI and IEE. All patients with upper gastrointestinal tumors enrolled in this study had received biopsies approximately

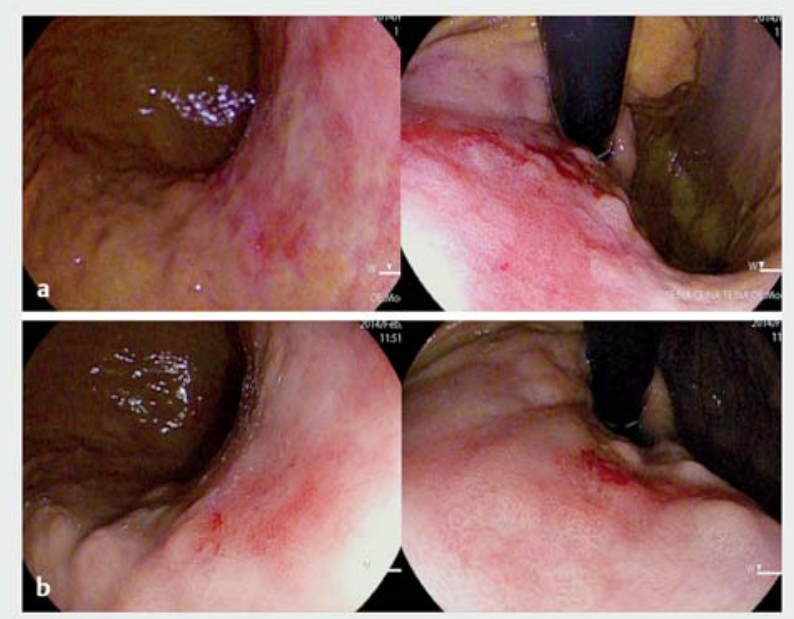

- Fig. 5 Endoscopic images from Case 5. EGC was observed as reddened lesions in the lesser curvature of the upper part of the gastric body only with WLI b. Lesions were not detected with IEE a, but were only observed with WLI $\mathbf{b}$, because of no difference between EGC and the surrounding red lesions in the intestinal metaplasia.

1 month before to the study. In most of them, it was difficult to appraise the post-biopsy scars, Therefore, we think that such scars did not have an influence on detection of EGC. In this study, IEE missed $20 \%$ of EGC lesions and WLI missed $30 \%$ of EGC lesions. We could not detect some lesions because they were very small although the entire gastric region was observed with screening endoscopy. When we could not find the gastric lesions, we never looked for the gastric region again. Therefore, we might not detect the gastric lesions at a higher rate.

In the current study, we investigated characteristics of detection factors related to use of IEE and WLI. An important detection factor using IEE was tumor circumference, where the rate of detection in the anterior wall and lesser curvature was significantly higher than in the posterior wall and greater curvature, determined using logistic regression analysis. The IEE system used in this study could brightly illuminate the gastric mucosa by means of strong light; however, in screening examinations, with IEE, gastric mucosa appeared slightly darker than 
when viewed with the WLI system. Because tumor circumference was not an important detection factor using WLI, the difference in detection rate as a result of tumor circumference using IEE was believed to have been caused by differences in brightness. Actually, when observing the dark area, we sometimes found it difficult to recognize gastric lesions located in a tangential direction or distant view; therefore, tumor circumference should be an important factor for detection of gastric cancer using IEE.

Color variation was an important detection factor when using WLI. Reddened or pale tumors were detected significantly more often than normal tumors using logistic regression analysis. The difference in color between normal gastric mucosa and EGC is an important detection factor in screening examinations. Using WLI, it was obvious that it was difficult to detect normal-colored tumors relative to reddened or pale tumors. In contrast, using IEE it was possible to detect normal-colored tumors, and the detection rate was $85.7 \%$. In our previous study, we demonstrated that IEE was significantly more effective in distinguishing the color difference between the normal epithelium and squamous cell carcinoma using the quantitative color difference method $\left(\Delta \mathrm{E}_{94}\right)$ [16]. Similarly, IEE is considered to enhance color differences between normal mucosa and normalcolored tumors; therefore, that modality may improve endoscopic detection and characterization of normal-colored EGC as compared with WLI.

In our study, only 3 cases of EGC in the lesser curvature were detected using IEE. IEE technology may lead to early detection of gastric cancer and determination of a demarcation line with a short-range view, such as the anterior wall and lesser curvature.

In some reddened tumors, we could easily distinguish between EGC and surrounding normal mucosa using WLI rather than using IEE. We found that 2 EGC cases were not detected using IEE, and could only be observed using WLI. The i-scan OE mode 2 has been designed to improve the contrast related to white-light observation by making the color tone of the overall image closer to that of the natural color [22]. Therefore, some cases involving red lesions in surrounding normal mucosa were overemphasized using IEE. In these cases, differences between EGC and surrounding mucosa could not be determined.

Limitations of this study were the small number of cases enrolled, and the fact that it was a single-institution study. In addition, inclusion of patients was limited to those having gastric or esophageal lesions. Consequently, there would have been selection bias regarding the participants. Further evidence is required from a larger-scale clinical trial of detection of EGC using IEE.

\section{Conclusion}

This pilot study demonstrated that the EGC detection rate without magnification was similar between the IEE and the WLI groups. However, IEE and WLI have different characteristics associated with detection of EGC. Our results suggest that EGC detection without magnification may be more effective if IEE and WLI systems were used in combination for screening examinations.

\section{Acknowledgments}

The authors thank Dr. Hisako Yoshida, Clinical research center of Saga University Hospital, for statistical advice.

\section{Competing interests}

None

References

[1] Muto M, Yao K, Kaise M et al. Magnifying endoscopy simple diagnostic algorithm for early gastric cancer (MESDA-G). Dig Endosc 2016; 28: 379-393

[2] Soetikno R, Kaltenbach T, Yeh R et al. Endoscopic mucosal resection for early cancers of the upper gastrointestinal tract. J Clin Oncol 2005; 23: $4490-4498$

[3] Ezoe $\mathrm{Y}$, Muto $\mathrm{M}$, Uedo $\mathrm{N}$ et al. Magnifying narrowband imaging is more accurate than conventional white-light imaging in diagnosis of gastric mucosal cancer. Gastroenterology 2011; 141: 2017 - 2025

[4] Maki S, Yao K, Nagahama T et al. Magnifying endoscopy with narrowband imaging is useful in the differential diagnosis between lowgrade adenoma and early cancer of superficial elevated gastric lesions. Gastric Cancer 2013; 16: 140 - 146

[5] Kato M, Kaise M, Yonezawa J et al. Magnifying endoscopy with narrow-band imaging achieves superior accuracy in the differential diagnosis of superficial gastric lesions identified with white-light endoscopy: a prospective study. Gastrointest Endosc 2010; 72 : $523-529$

[6] Tajiri H, Niwa H. Recent Advances in Electronic Endoscopes: Imageenhanced endoscopy. JMAJ 2008; 51: 199-203

[7] Muto M, Minashi K, Yano T et al. Early detection of superficial squamous cell carcinoma in the head and neck region and esophagus by narrow band imaging: a multicenter randomized controlled trial. J Clin Oncol 2010; 28: 1566 - 1572

[8] Yao K, Doyama H, Gotoda T et al. Diagnostic performance and limitations of magnifying narrow-band imaging in screening endoscopy of early gastric cancer: a prospective multicenter feasibility study. Gastric Cancer 2014; 17: 669-679

[9] Yao K, Anagnostopoulos GK, Ragunath K. Magnifying endoscopy for diagnosing and delineating early gastric cancer. Endoscopy 2009; 41: $462-467$

[10] Yao K, Iwashita A, Tanabe H et al. Novel zoom endoscopy technique for diagnosis of small flat gastric cancer: a prospective, blind study. Clin Gastroenterol Hepatol 2007; 5: 869-878

[11] Subramanian V, Ragunath K. Advanced endoscopic imaging: a review of commercially available technologies. Clin Gastroenterol Hepatol 2014; 12: $368-376 . e 1$

[12] Horimatsu T, Sano Y, Tanaka S et al. Next-generation narrow band imaging system for colonic polyp detection: a prospective multicenter randomized trial. Int J Colorectal Dis 2015; 30: 947-954

[13] Osawa H, Yamamoto H. Present and future status of flexible spectral imaging color enhancement and blue laser imaging technology. Dig Endosc 2014; 26: 105 - 115

[14] Kaneko K, Oono Y, Yano T et al. Effect of novel bright image enhanced endoscopy using blue laser imaging (BLI). Endosc Int Open 2014; 2: E212-E219

[15] Yoshida N, Yagi N, Inada Y et al. Ability of a novel blue laser imaging system for the diagnosis of colorectal polyps. Dig Endosc 2014; 26: $250-258$ 
[16] Kodashima S, Fujishiro M, Ono S et al. Evaluation of a new image-enhanced endoscopic technology using band-limited light for detection of esophageal squamous cell carcinoma. Dig Endosc 2014; 26: 164 171

[17] Sano Y, Ikematsu H, Fu KI et al. Meshed capillary vessels by use of narrow-band imaging for differential diagnosis of small colorectal polyps. Gastrointest Endosc 2009; 69: 278-283

[18] Uedo N, Ishihara R, lishi $\mathrm{H}$ et al. A new method of diagnosing gastric intestinal metaplasia: narrow-band imaging with magnifying endoscopy. Endoscopy 2006; 38: 819-824

[19] Kuznetsov K, Lambert R, Rey JF. Narrow-band imaging: potential and limitations. Endoscopy 2006; 38: 76-81
[20] Miyaki R, Yoshida S, Tanaka S et al. A computer system to be used with laser-based endoscopy for quantitative diagnosis of early gastric cancer. J Clin Gastroenterol 2015; 49: 108-115

[21] Neumann H, Fujishiro M, Wilcox CM et al. Present and future perspectives of virtual chromoendoscopy with i-scan and optical enhancement technology. Dig Endosc 2014; 26: 43 - 51

[22] Tsuji Y, Ohata K, Sekiguchi M et al. Magnifying endoscopy with narrow-band imaging helps determine the management of gastric adenomas. Gastric Cancer 2012; 15: 414-418

[23] Yao K, Anagnostopoulos GK, Ragunath K. Magnifying endoscopy for diagnosing and delineating early gastric cancer. Endoscopy 2009; 41: $462-467$

\section{CORRECTION}

Daisuke Yamaguchi, Shinya Kodashima, Mitsuhiro Fujishiro et al. Evaluation of image-enhanced endoscopic technology using advanced diagnostic endoscopy for the detection of early gastric cancer: a pilot study.

Endoscopy International Open 2017.

DOI: $10.1055 / \mathrm{s}-0043-113632$

The order of $\mathbf{F i g} .1$ was corrected. This was corrected in the online version on September 21, 2017. 